\title{
Cyclotron braid group approach to
}

\section{Laughlin correlations}

\author{
Janusz Jacak ${ }^{1}$, Ireneusz Jóźwiak ${ }^{2}$, Lucjan Jacak ${ }^{1 *}$ and \\ Konrad Wieczorek ${ }^{1}$ \\ ${ }^{1}$ Institute of Physics, Wrocław University of Technology, \\ Wyb. Wyspiańskiego 27, 50-370 Wrocław, Poland \\ janusz.jacak@pwr.wroc.pl; lucjan.jacak@pwr.wroc.pl; \\ konrad.wieczorek@pwr.wroc.pl \\ ${ }^{2}$ Institute of Informatics, Wrocław University of Technology, \\ Wyb. Wyspiańskiego 27, 50-370 Wrocław, Poland \\ ireneusz.jozwiak@pwr.wroc.pl
}

\begin{abstract}
Homotopy braid group description including cyclotron motion of charged interacting two-dimensional (2D) particles at strong magnetic field presence is developed in order to explain, in algebraic topology terms, Laughlin correlations in fractional quantum Hall systems. There are introduced special cyclotron braid subgroups of a full braid group with 1D unitary representations suitable to satisfy Laughlin correlation requirements. In this way an implementation of composite fermions (fermions with auxiliary flux quanta attached in order to reproduce Laughlin correlations) is formulated within uniform for all 2D particles braid group approach. The fictitious fluxes — vortices attached to the composite fermions in a traditional formulation are replaced with additional cyclotron trajectory loops unavoidably occurring when ordinary cyclotron radius is too short in comparison to particle separation and does not allow for particle interchanges along single-loop cyclotron braids.
\end{abstract}

e-print archive: http://lanl.arXiv.org/abs/0910.4250 
Additional loops enhance the effective cyclotron radius and restore particle interchanges. A new type of $2 \mathrm{D}$ particles - composite anyons is also defined via unitary representations of cyclotron braid subgroups. It is demonstrated that composite fermions and composite anyons are rightful 2D particles, not auxiliary compositions with fictitious fluxes and are associated with cyclotron braid subgroups instead of the full braid group, which may open also a new opportunity for non-Abelian composite anyons for topological quantum information processing applications, due to richer representations of subgroup than of a group.

\section{Introduction}

Specific topological properties of two-dimensional (2D) $N$-particle systems have been recognized within algebraic topology [1] using homotopy group methods $[2,3]$. They turned out to be of particular significance in understanding of quantum behavior of 2D electron systems widely experimentally investigated in Hall configuration since the discovery of fractional quantum Hall effect (FQHE) [4]. A hierarchy of Landau level (LL) fractional fillings was observed [4-6] and explained by new topological concepts closely related with planar geometry [7-9]. The exceptional topology of 2D systems is connected with nontrivial homotopy groups [2,11-14] describing planar trajectories for many-particle systems. Classes of topologically nonequivalent closed loops in the configuration space of a system of $N$ identical particles build up $\pi_{1}$ homotopy group [1], called in this case a braid group (full and pure for indistinguishable and distinguishable particles, respectively) [2,11-13]. Braid groups for 2D case are infinite, while for higher dimensions are finite and equal (full braid groups) to the permutation group $S_{N}$ (for 1D case this formalism is irrelevant) [3]. Unitary representations, in particular one-dimensional (1DURs) of the full braid group serve for identification of quantum particles corresponding to the same classical ones [9]. For $S_{N}$ there exist only two distinct 1DURs: $\sigma_{i} \rightarrow e^{i \pi}$ or $\sigma_{i} \rightarrow e^{i 0},\left(\sigma_{i}-\right.$ the interchange of $i$ th and $(i+1)$ th particles) corresponding to fermions and bosons, respectively. In 2D there is, however, an abundance of distinct 1DURs of the full braid groups: $e^{i \theta}, \theta \in(-\pi, \pi]$, associated with anyons (Abelian) $[5,7-9,15]$. Anyons reveal a fractional statistics - the interchange of two anyons results in a $\theta$ phase shift of the wave function $[9,10]$.

Crucial for understanding of FQHE was the formulation by Laughlin $[7,8]$ of the wave function for a ground state of $2 \mathrm{D}$-charged particle system at strong magnetic field presence. The Laughlin function [8] corresponds to $\frac{1}{p}$ ( $p$-odd integer) fractional filling of the lowest LL and is a generalization of the Slater determinant. The Slater function for completely filled lowest LL, for magnetic field in cylindrical gauge, has the form (up to an exponential factor) of the Vandermonde determinant, $\prod_{i<j}\left(z_{i}-z_{j}\right), z_{i}=x_{i}+i y_{i}$ stands here 
for $i$ th 2D particle coordinate expressed as a complex number. Replacement in this Slater function of the Vandermonde determinant with the Jastrow polynomial, $\prod_{i<j}\left(z_{i}-z_{j}\right)^{p}$, ( $p$ odd integer), results in the Laughlin wave function $[7,8]$ for filling $\frac{1}{p}$. The Laughlin function is still antisymmetric but differs from the Slater function in the phase shift acquired due to interchange of a 2D particle pair. For the Vandermonde function it is $\pi$, while for the Jastrow function $p \pi$. The difference in phases is important in planar geometry (in higher dimensions the phase shift has no meaning), but $2 \pi$ periodicity of the phase factor, $e^{i p \pi}=e^{i \pi}$, also in 2D seemingly does not allow for distinguishing of the statistics imposed by Laughlin correlations from ordinary fermion antisymmetry. Therefore more subtle topological attitude - the braid group methods, should be here applied in order to grasp the novelty introduced by the Laughlin function.

The phenomenological approach to Laughlin correlations was introduced in terms of composite fermions ( $\mathrm{CFs}$ ), regarded as ordinary $2 \mathrm{D}$ fermions with associated to each particle even number of magnetic flux quanta $[16,17]$. The even number, $q$, of magnetic flux quanta attached to individual particles does not change antisymmetry of the total system wave function, but due to Aharonov-Bohm effect results in additional $q \pi$ phase shift during particle pair interchange [9]. In this manner the magnetic field local fluxes, called as vortices, attached to CFs model the Laughlin correlations $[7,8]$. The $\mathrm{CF}$ attitude suffers, however, from an artificial character of the construction, i.e., not explained source of the magnetic field fluxes changing fermions into CFs.

Nevertheless, CFs regarded as only weakly (residually) interacting, surprisingly well describe Hall systems $[16,17]$ especially within the lowest LL (for higher LLs the inter-level mixing effects perturb a CF picture). The vortices of CFs, oriented oppositely to the external field are assumed to be able to screen the external magnetic field, and in the effective weaker resultant field, one can deal with an integer quantum Hall effect - which yields the fractional hierarchy $\frac{p}{2 p \pm 1}[16,17]$. In other words, the oscillations in Hall conductivity (FQHE) can be associated with Szubnikov-de Haas oscillations in an effective reduced magnetic field. The interesting observation supporting this model is the so-called Hall metal state [18] at filling fraction $\frac{1}{2}$, when the total external magnetic field should be canceled by the averaged internal field of $\mathrm{CF}$ fluxes. It still arises, however, an important question of what is the physical source of these magnetic flux quanta, i.e., vortices, attached to charged particles which alter original fermions into CFs and how to understand localization of magnetic field fluxes on individual particles.

In the present paper, we demonstrate the braid structure of CFs, as particles with statistical properties required by Laughlin correlations, via association them with cyclotron braid subgroups instead of the full braid groups. Introduced below cyclotron braid subgroups reflect the classical 
braid picture for 2D $N$-particle charged system at the presence of magnetic field. The quantization, via 1DURs of these cyclotron subgroups, allow for natural explanation of Laughlin correlations, without invoking artificial vortices. In particular, this approach elucidates the CF construction and the true character of auxiliary Jain's vortices [16], which turned out a useful model of basic trajectory loops unavoidably occurring on cyclotron braids at fractional LL fillings $\frac{1}{p}$. The multi-loop braids from cyclotron braid subgroups allow for particle interchange in the braid picture, when the singleloop cyclotron diameter is shorter than the particle separation, which precludes their exchanges along single-loop cyclotron trajectories. In order to enhance cyclotron radius and to restore particle interchanges in braid picture, each particle must traverse, in classical braid meaning, a closed $p$-loop cyclotron trajectory, or in quantum language, each particle takes away $p$ quanta of the external magnetic field flux; $p-1$ of them play the equivalent role as $p-1$ flux quanta attached to each $\mathrm{CF}$ in a traditional model, reducing the external field. Topological implementation of CFs in braid group terms was not previously formulated due to periodicity of 1DURs. Association of composite particles (including CFs) with a separate cyclotron braid subgroups allows, however, for distinguishing them in terms of unitary representations, despite $2 \pi$ periodicity of the unitary factor.

The paper is organized as follows. In the next paragraph the main lines of the braid group approach to quantum systems are summarized. In the following one, the original idea of cyclotron braid subgroups is developed and applied to description of CFs, and more generally to composite-anyons. The multi-loop structure of cyclotron braids, essential for CF description, is explained. The role of the Coulomb interaction is described in a separate paragraph. The possible application of introduced composite anyons to topological quantum information processing is indicated.

\section{Braid group method for description of $N$-particle systems}

\subsection{Definitions of a full and a pure braid groups}

Braid group is a first homotopy group [1], $\pi_{1}$, for configuration space of $N$-particle system. $\pi_{1}(A)$ is a group of topologically nonequivalent classes of closed trajectories in the space A. In the case of $N$-particle system, $A$ is an appropriate classical configuration space. The braid groups display only a possible classical motion of $N$-particle system and a quantization is performed via unitary representations of classical braid trajectories, as it is described below. 
The configuration space of $N$ identical particles located on a manifold $M$ (e.g., $R^{n}$, or compact manifolds) is defined as: $Q_{N}(M)=\left(M^{N} \backslash \Delta\right) / S_{N}$, for indistinguishable identical particles, and as: $F_{N}(M)=M^{N} \backslash \Delta$, for distinguishable identical particles; $M^{N}$ is the $N$ th Cartesian product of the manifold $M, \Delta$ is the set of diagonal points (when coordinates of two or more particles coincide), subtracted in order to preserve conservation of the particle number, $S_{N}$ is the permutation group - the quotient structure is introduced in order to account for indistinguishability of quantum particles. Note, that indistinguishability of particles is here artificially introduced in the definition of configuration space, which indicates that this property is independent of quantum uncertainty principles.

For these configuration spaces two types of braid groups are defined [2]:

$$
\left.\pi_{1}\left(Q_{N}(M)\right)=\pi_{1}\left(M^{N} \backslash \Delta\right) / S_{N}\right),
$$

a full braid group and

$$
\pi_{1}\left(F_{N}(M)\right)=\pi_{1}\left(M^{N} \backslash \Delta\right),
$$

a pure braid group.

For $M=R^{n}, n>2$ the braid group have a simple structure. The full braid group, for $n>2$, equals to a permutation group $S_{N}$ (note, that this group is a finite group, of rank $N$ !). For $M=R^{2}$ (and for compact locally $2 \mathrm{D}$ manifolds, as a sphere or a torus in $3 \mathrm{D}$ ) the braid groups are infinite highly nontrivial groups.

It is convenient to illustrate a structure of the braid groups for the plane via a simple presentation using geometrical braids $[2,12]$ — cf. Figure 1. In this figure there are depicted: (a) geometrical braid corresponding to the generator $\sigma_{i}$ of the full braid group (interchange of the $i$ th and $(i+1)$ th strings representing particle trajectories), (b) geometrical braid corresponding to the inverse element of the generator, $\sigma_{i}^{-1},(\mathrm{c})$ geometrical braid for the square of the generator $\left(\sigma_{i}\right)^{2} \neq e$ ( $e-$ the neutral element of the group). In

(a)

(b)

(c)
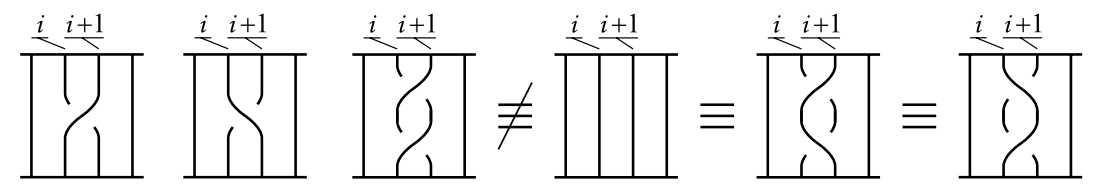

Figure 1: Geometrical braid presentation for $B_{N}$ : (a) the generator $\sigma_{i}$; and (b) its inverse, $\sigma_{i}^{-1}$; (c) square of the generator $\sigma_{i}^{2}$. 
3D $\left(\sigma_{i}\right)^{2}=e$, which simplifies the braid structure to ordinary permutation group $S_{N}$, while in $2 \mathrm{D}\left(\sigma_{i}\right)^{2} \neq e$ and it causes complicated (of infinite type) structure of planar braids.

One can list formal conditions imposed on generators $\sigma_{i}, i=1, \ldots, N-1$, in order to define the full braid group for the plane, in an abstract manner $[2,11]$. These conditions are written below,

$$
\begin{aligned}
\sigma_{i} \sigma_{i+1} \sigma_{i} & =\sigma_{i+1} \sigma_{i} \sigma_{i+1}, \text { for } 1 \leq i \leq N-2 \\
\sigma_{i} \sigma_{j} & =\sigma_{j} \sigma_{i} . \text { for } 1 \leq i, j \leq N-1,|i-j| \geq 2
\end{aligned}
$$

The initial ordering of particles is not conserved for braids from a full braid group, while for braids from a pure one, the ordering must be conserved. The generators $l_{i j}$ of a pure braid group [2] correspond to double exchanges of particle pairs, $i j$, however, without any perturbation of the assumed ordering of particles, and have the following form in terms of $\sigma_{i}$ generators:

$$
l_{i j}=\sigma_{j-1} \cdot \sigma_{j-2} \cdots \sigma_{i+1} \cdot \sigma_{i}^{2} \cdot \sigma_{i+1}^{-1} \cdots \sigma_{j-2}^{-1} \cdot \sigma_{j-1}^{-1}, \quad 1 \geq i \geq j \geq N-1 .
$$

The pure group is a subgroup of the full group since the generators $l_{i j}$ are expressed by means of $\sigma_{i}$ generators. For defining relations for generators of the pure group cf. $[2,12]$.

Note that the connection between the full braid group and the pure one is given by the quotient relation [2], $B_{N} / \pi_{1}\left(F_{N}\left(R^{2}\right)\right)=S_{N}\left(B_{N}\right.$ stands here for commonly used notation for the full braid group for the plane) [11].

For the sphere $S^{2}$ the additional condition for generators, beyond those given by equations (2.3) and (2.4), is imposed [2],

$$
\sigma_{1} \cdot \sigma_{2} \cdots \sigma_{n-2} \cdot \sigma_{n-1}^{2} \cdot \sigma_{n-2} \cdots \sigma_{2} \cdot \sigma_{1}=e
$$

which displays the fact that on the sphere a loop of a selected particle embracing all other particles is contractible to a point. For the torus $T$ additional relations [14] correspond to two nonequivalent paths of each particle on this not simple-connected manifold.

\subsection{Quantization in braid group picture}

Quantization of the system of $N$ identical indistinguishable particles can be performed by application of the Feynman integral over trajectories, leading 
to a propagator (probability for a transition from a point $a$ to a point $b$ in the configuration space):

$$
I_{a \rightarrow b}=\int d \lambda e^{i S\left[\lambda_{a, b}\right] / \hbar},
$$

where $S\left[\lambda_{a, b}\right]$ is the classical action for the trajectory $\lambda_{a, b}$ in the classical configuration space of $N$-particle system, $d \lambda$ is a measure in a trajectory space. To each trajectory linking $a$ and $b$ points in the $N$-particle configuration space, one can attach, however, additional closed loops which are elements of the full braid group. Thus resulting trajectories fall into separated topologically nonequivalent classes, represented by elements of the full braid group. Therefore an additional unitary factor (the weight of the separated trajectory class) should be added $[9,10]$ in the formula for integration over trajectories, together with the additional sum over the braid group elements (since each element of the full braid group can be attached to a loop-less simple trajectory $\lambda_{a, b}$ ):

$$
I_{a \rightarrow b}=\sum_{l \in \pi_{1}} e^{i \alpha_{l}} \int d \lambda_{l} e^{i S\left[\lambda_{l(a, b)]}\right] / \hbar},
$$

$\pi_{1}$ represents here the full braid group. These factors $e^{i \alpha_{l}}$ form a 1DUR of the full braid group. Distinct representations correspond to distinct types of quantum particles, linked to the same classical ones.

As was mentioned in the Introduction section, for $S_{N}$, which is the full braid group for $3 \mathrm{D}$ manifolds (and for higher dimensions), there exist only two distinct 1DURs,

$$
\sigma_{i} \rightarrow\left\{\begin{array}{l}
e^{i 0}, \\
e^{i \pi},
\end{array}\right.
$$

corresponding to bosons and fermions, respectively (leading to a symmetry and antisymmetry properties of relevant wave functions). For 2D space (the plane), the braid group (considerably richer than $S_{N}$ ) has an infinite number of 1DURs [12,13], written for the group generators as $\sigma_{i} \rightarrow e^{i \theta}, \theta \in(-\pi, \pi]$, where each $\theta$ enumerates a different type of so-called anyons $[5,7-10,15]$. Note that elements of 1DUR of the full braid group do not depend on the index $i$ (of the generator $\sigma_{i}$ ) owing to condition (2.3) imposed on generators. Because the 1DUR elements commute, then from equation (2.3) it follows that $e^{i \theta_{i}}=e^{i \theta_{i+1}}$, where $\sigma_{i} \rightarrow e^{i \theta_{i}}$, which gives this $i$-index independence of 1DUR elements. 
For the sphere $S^{2}$ 1DURs have the form [12,13], $e^{i \theta}$, where $\theta=k \pi /(N-1)$, $k=0,1,2, \ldots, 2 N-3$. It is interesting to notice, that for two particles on the sphere (i.e., for $N=2$ one has only $k=0,1$ ) only bosonic or fermionic statistics are available (actually because of equation (2.6)), and anyons may occur on the sphere for three particles, at least. In the case of a torus $T$, for an arbitrary number $N$ of particles, $\theta=0$ or $\pi$ are admitted only $[13,14]$ thus on a torus any anyons do not exist, except for fermions and bosons. This result was generalized [13] also for all compact locally 2D manifolds with exception for the sphere.

The classical trajectories from the full braid group have no quantum meaning. Quantum particles do not traverse any braid trajectories since they do not have trajectories at all. In agreement with the general rules of quantization $[19,20], N$-particle wave function must transform according to 1DUR of an appropriate element of the braid group when the particles traverse classically a closed loop in the configuration space of $N$-particle system corresponding to this braid element. As braids from the full braid group describe interchanges of particles, thus corresponding 1DURs display statistics phase factors.

Note that important are also multi-dimensional unitary irreducible representations (MDURs) of braid groups. According to an idea of Kitaev $[15,21]$, an arbitrary unitary evolution of multi-qubit system (e.g., of a double qubit gate for QIP) $[15,22]$ can be approximated by an MDUR (of an appropriate rank) of a full braid group, provided the sufficient density level of MDURs in the unitary matrix space [15]. MDURs can be linked with degenerated low-energy excitations (quasiparticles/quasiholes, typically regarded as anyons) above the ground state for some fractional LL fillings. Since elements of MDUR do not commute, as matrices, these degenerate states of anyons are referred as non-Abelian anyons [15]. Unfortunately, the non-Abelian anyons recently investigated in particular low excited states for $5 / 2$ and $12 / 5$ LL filling factors correspond probably to not sufficiently dense MDURs (for non-Abelian anyons in $\frac{5}{2}$ case the MDURs are not dense enough to approximate needed qubit gates [15], and another considered now state $\frac{12}{5}$ is still disputable [23]). Thus searching for other opportunities for fractional statistics systems with more dense MDURs associated with non-Abelian anyons is of high significance. In the next section, we will introduce a cyclotron braid subgroups of a full braid group. As subgroups have usually richer representations than a group, thus one can expect that the cyclotron braid subgroups would be convenient for topology methods for QIP, since the relevant MDURs of cyclotron subgroups would be more dense in comparison to representations of a full braid group. 


\section{Cyclotron braid groups at magnetic field presence}

Let us emphasize that the braid groups described above are constructed in the absence of the magnetic field. Elements of the full braid group were all trajectories without any modifications caused by the magnetic field. Inclusion of the magnetic field considerably confines, however, the variety of admitted trajectories. All trajectories must be of cyclotron shape at the presence of the magnetic field and this property highly modifies the braid group structure. Instead of a full braid group, cyclotron trajectories form a braid subgroup - a cyclotron subgroup, in particular at $1 / p$ fractional LL filling. It leads to an opportunity for an implementation of CFs (2D particles at a strong magnetic field presence) via cyclotron subgroups of the full braid group. Following this idea, at magnetic field presence the summation in the Feynman propagator must be confined to the subgroup elements only, i.e., to selected, suitably to cyclotron motion, classes of trajectories instead of arbitrary elements of the full braid group. The 1DURs of the cyclotron braid subgroups will thus substitute the 1DURs of the full braid group in the path integral (2.8).

Let us consider 2D-charged particle system with planar density $\frac{N}{S}(N$ is the number of particles, $S$ is the surface of a sample) and at presence of a perpendicular magnetic field $B$. Topology of a manifold where the particles are located is assumed here the same as of the plane $R^{2}$ (it would be considered as an compact subset of $R^{2}$, without a boundary) [2]. For this manifold one can define the full braid group [2,11-13], being the $\pi_{1}$ homotopy group [1] of the configuration space for $N$ indistinguishable particles on $R^{2}$. This braid group is commonly called as $B_{N}$ (the Artin group) [11] and is generated by interchanges of neighboring particles at chosen their ordering $[2,12]$,

$$
\sigma_{i}, \quad i=1, \ldots, N-1 \text {, }
$$

with defining relations given by equations (2.3) and (2.4).

\subsection{Definition of the cyclotron braid subgroup}

Let us define the cyclotron braid subgroup by means of its generators $b_{i}^{(p)}$ of the following form:

$$
b_{i}^{(p)}=\sigma_{i}^{p}, \quad p=1,3,5,7,9, \ldots ; \quad i=1, \ldots, N-1,
$$

where each $p$ corresponds to a different type of the cyclotron subgroup and $\sigma_{i}$ are generators of the full braid group. 
(a)

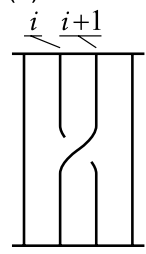

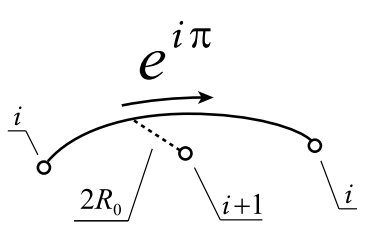

(b)

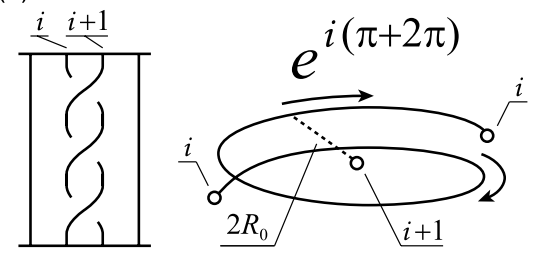

Figure 2: (a) The generator $\sigma_{i}$ of the full braid group and the corresponding relative trajectory of particle $i$ th and $(i+1)$ th exchange; $(\mathrm{b})$ the generator of the cyclotron braid subgroup, $b_{i}^{(p)}=\sigma_{i}^{p}$ (in the figure $p=3$ ), corresponds to additional $\frac{p-1}{2}$ loops when the $i$ th particle interchanges with the $(i+$ 1 )th one (an additional loop results in $2 \pi$ phase shift; $2 R_{0}$ — inter-particle separation).

The full braid group element $b_{i}^{(p)}$ (the generator of the cyclotron braid subgroup of type $p$ ) represents the interchange of $i$ th and $(i+1)$ th particles with $\frac{p-1}{2}$ loops. It is clear due to the definition of the single interchanges by the generators $\sigma_{i}$ of the full braid group, cf. Figure 2.

The generators $b_{i}^{(p)}$ create the subgroup of the full braid group as they are expressed by generators $\sigma_{i}$ of the full braid group. The $b_{i}^{(p)}$ do not, however, satisfy condition (2.3) (cf. Figure 3(c)), while condition (2.4) is maintained for $b_{i}^{(p)}: b_{i}^{(p)} b_{j}^{(p)}=b_{j}^{(p)} b_{i}^{(p)}$, for $1 \leq i, j \leq N-1, \quad|i-j| \geq 2(\mathrm{cf}$. Figure $3(\mathrm{~d}))$.

Condition (2.3) resulted in independence of 1DUR of the braid group generator index $i$. Disappearance of this condition for the cyclotron braid subgroup leads to possible dependence of the subgroup 1DUR on the index $i$, in general. The 1DURs of the full group, $\sigma_{i} \rightarrow e^{i \alpha}$, confined to the subgroup, do not depend, however, on $i$ and yield:

$$
b_{i}^{(p)} \rightarrow e^{i p \alpha},
$$

$p$ an odd integer, $\alpha \in(-\pi, \pi]$. These 1DURs of the cyclotron braid subgroup, numbered by the pairs $(p, \alpha)$, describe composite anyons, and, in a particular case, CFs for $\alpha=\pi$.

\subsection{Multi-loop cyclotron braid structure}

For the above construction of the cyclotron subgroups the $N$-particle wave function acquires an appropriate phase shift due to a peculiar type of particle 
(a)
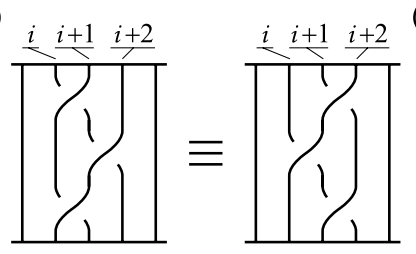

(c)

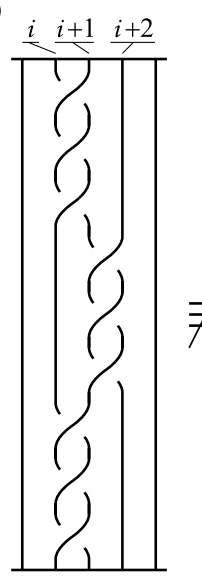

(b)
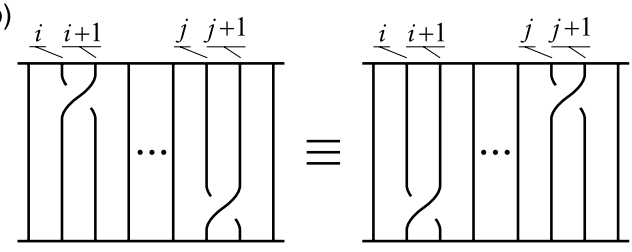

(d)
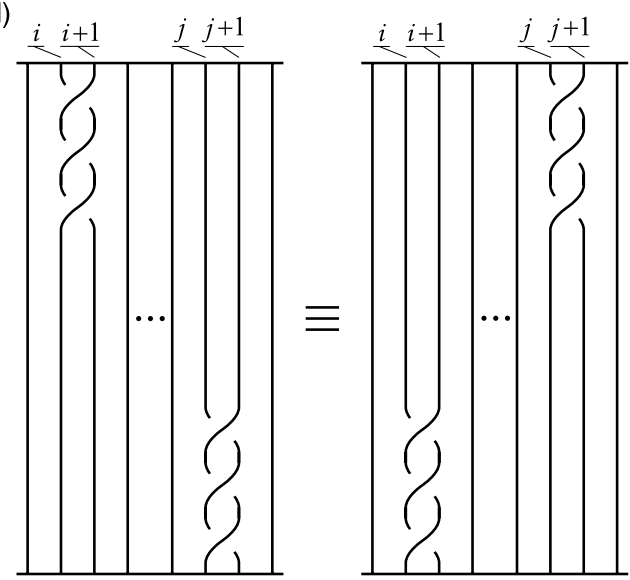

Figure 3: Formal conditions defining a full braid group for $R^{2}$, cf. equations (2.3) and (2.4); violation of condition (2.3) for the cyclotron subgroup generators $b_{i}^{(3)}$ (c) (condition (2.4) is maintained for the cyclotron subgroup generators $(d))$.

interchanges in the braid picture, i.e., we replace the Aharonov-Bohm phase of fictitious fluxes by additional braid loops (each loop adds $2 \pi$ to the total phase shift - cf. Figure 2). It is noticeable if one takes into account the rules of quantization in the braid group framework $[19,20]$. In agreement with them, $N$-particle wave function must transform according to $1 \mathrm{DUR}$ of an appropriate element of the braid group, when the particles traverse classically a closed loop in the configuration space of $N$-particle system corresponding to this braid element. For the cyclotron braid subgroup generated by $b_{i}^{(p)}, i=1, \ldots, N-1$ (defined by equation (3.2)), we obtain for particle pair interchange the total wave function phase shifts $p \pi$ (for $\alpha=\pi$ in the representation given by equation (3.3)), as is required by Laughlin correlations $[7,8]$, without modeling them by fictitious vortices.

\subsection{Definition of an individual particle cyclotron trajectory}

Note, that each additional loop of a relative trajectory for particle pair interchange (such a trajectory is needed for definition of the subgroup 
(a)

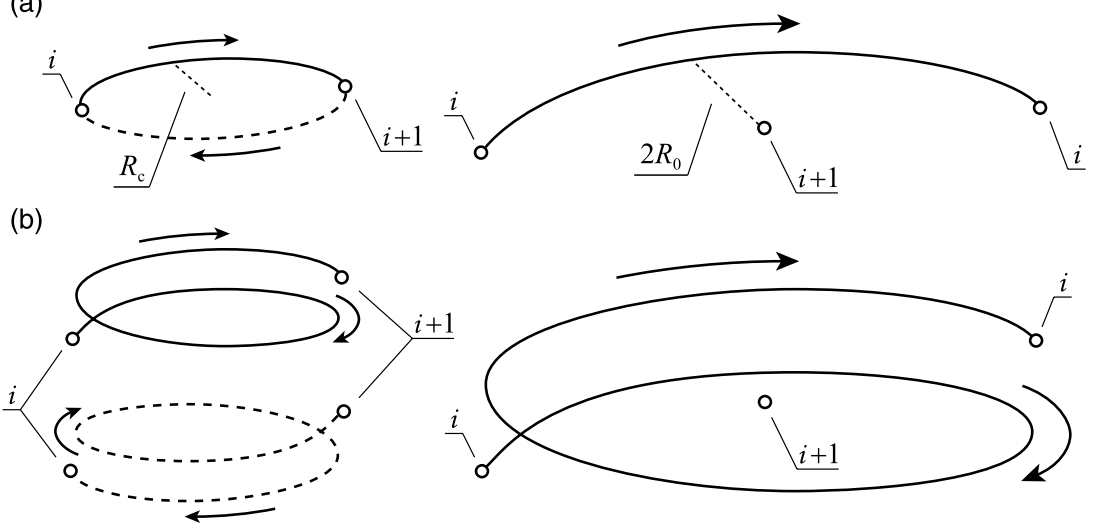

Figure 4: Cyclotron (left) and corresponding relative (right) trajectories for interchanges of $i$ th and $(i+1)$ th 2D-particles at strong magnetic field, (a) for $\nu=1$; (b) for $\nu=\frac{1}{3}$, respectively (3D for better visualization); in both cases, $\nu=1, \frac{1}{3}$, the appropriate cyclotron radius $R_{\mathrm{c}}$ fits with the inter-particle separation $2 R_{0}=2 R_{\mathrm{c}}, 2 R_{0}$ - inter-particle separation is fixed by the Coulomb repulsion.

generators $\left.b_{i}^{(p)}\right)$ reproduces an additional loop in individual cyclotron trajectories for both interchanging particles - cf. Figure 4. In this figure, the cyclotron motion of particle pair is depicted for the interchange of $i$ th and $(i+1)$ th particles separated by double cyclotron radius $2 R_{\mathrm{c}}$, without any additional loops (a) and with the additional loop (b), respectively. The cyclotron trajectories are repeated in the relative trajectory (right) with a double radius in comparison to the individual particle trajectories (left). In quantum language, with regard to classical multi-loop cyclotron trajectories, one can conclude only on the number, $\frac{B S}{N} / \frac{h c}{e}$, of flux quanta per single particle in the system, which for the LL filling $\frac{1}{p}$ is $p$, i.e., the same as the number of cyclotron loops of each particle. Thus a simple pictorial rule could be here formulated: an additional loop on a braid corresponding to particle interchange, introduced in accordance with generators of the cyclotron braid subgroup, results in two additional flux quanta piercing the individual particle cyclotron trajectories. It immediately follows from the definition of the cyclotron trajectory.

One can define this trajectory as the individual particle trajectory corresponding to a double interchange of the particle pair (cf. Figure 5). In this way, the cyclotron trajectories of both interchanging particles are closed, similarly as closed the relative trajectory for double interchange is. If the interchange is simple, i.e., without any additional loops, the corresponding individual particle cyclotron trajectories are also simple, single-loop 
(a)

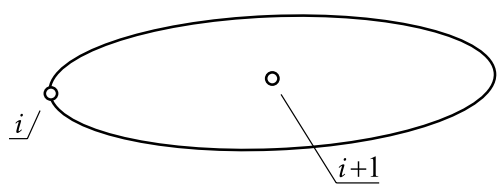

(b)

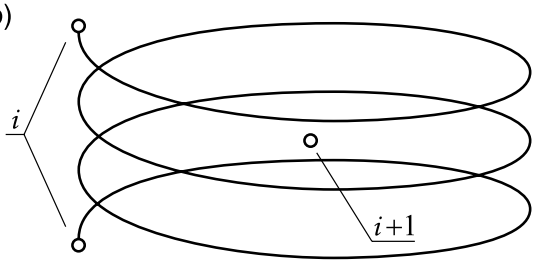

Figure 5: Individual particle closed cyclotron trajectories corresponding to double relative trajectories for interchanges of $i$ th and $(i+1)$ th $2 \mathrm{D}$-particles at strong magnetic field, (a) for $\nu=1$; (b) for $\nu=\frac{1}{3}$, respectively (3D for better visualization); the number of $B$ field flux quanta per particle is indicated in both cases, $\nu=1, \frac{1}{3}$; the resulting cyclotron radius $R_{\mathrm{c}}$ fits with the inter-particle separation $2 R_{0}=2 R_{\mathrm{c}}$ in both cases.

(circles on 2D plane). But when the interchange of particles is multi-loop, as associated with $p$-type cyclotron subgroup $(p>1)$, the double interchange relative trajectory has $2 \frac{p-1}{2}+1$ closed loops and the individual cyclotron trajectories are also multi-loop, with $p$ loops. It is illustrated in Figure 5.

It is worth to emphasize the difference between turns of windings (e.g., of a wire) and multi-loop 2D cyclotron trajectories. The latter ones cannot enhance a piercing total magnetic field flux $B S$ (thus the number of flux quanta per particle coincides with the number of loops of closed cyclotron individual particle trajectory), while in the former case, each turn of windings adds a new portion of the flux as a new turn adds a new surface in fact (which is no case in $2 \mathrm{D}$ ).

\subsection{Relation of cyclotron braid subgroups with CFs}

We will explain below that the multi-loop shape of the relative trajectory for interchanges, as defined by the subgroup generators (3.2) (and corresponding multi-loop form of individual particle cyclotron trajectories), is an unavoidable property in the case when inter-particle separation (resulted from the density $\frac{N}{S}$ and fixed by the Coulomb repulsion) is greater than the double value of single-loop cyclotron radius. In this case, in particular at $\frac{1}{p}$ LL filling fraction, any exchanges along simple single-loop cyclotron trajectories are impossible, because the corresponding cyclotron radius is too short. In order to restore a possibility of particle interchanges (necessary, on the other hand, for braid structure definition and thus for statistics determination), too short cyclotron radius must be enhanced. The way to enhance the effective cyclotron radius, which would again fit to inter-particle separation, is the multi-loop character of cyclotron motion and simultaneously resulting multi-loop braids for particle interchanges (represented by generators of the 
cyclotron braid subgroups, equation (3.2)). The additional cyclotron loops take away a part of the external field flux and thus reduce the effective field which leads to an expected growth of a resulting cyclotron radius.

The total flux of the external field through the surface $S$ is $B S$. For $p$ type of CFs, if one considers the relative trajectory of double interchange of $i$ th and $(i+1)$ th particles (thus closed and with $2 \frac{p-1}{2}+1=p$ loops), one gets the individual particle closed cyclotron trajectories with the same number $p$ of loops (cf. Figure 5), embracing the total flux $p \frac{h c}{e}$ (each loop takes away a single flux quantum in accordance with the above presented interpretation). Thus for $p$ type of CFs we deal with closed $p$-loop cyclotron trajectories of particles, i.e., $p$ flux quanta per particle, $B S=N p \frac{h c}{e}$. On the other hand, the degeneracy of the LL equals to $N_{0}=\frac{S B e}{h c}$, (neglecting spin) and for fractional filling $\nu, N_{0}=\frac{N}{\nu} \cdot \frac{B S}{N}=\frac{h c}{e} \frac{1}{\nu}$ gives $\frac{1}{\nu}$ flux quanta per particle, which fits with the previous estimation only for $\nu=\frac{1}{p}$.

In the case of $p$-loop trajectory each loop has its size adjusted to the external magnetic field flux diminished by $p-1$ quanta per particle taken away by remaining loops, exactly as in the case of the Jain's model. Indeed, if $B S=\frac{h c}{e} p N$, then $\frac{h c}{e}=\frac{B}{p} \frac{S}{N}$ and $\frac{S}{N}$ corresponds to $p$ times lowered field. Following an analogy with Jain's model, one could argue that for $\nu=\frac{1}{2}$ and $p=3$, two loops per particle take away the total $B$ field flux and the third loop has to be of infinite radius (Hall metal [18]) for zero rest-field.

The additional loops take away flux quanta simultaneously diminishing the field; this gives an explanation of the fictitious Jain fluxes screening the field $B$. Thus the presented cyclotron subgroup implementation of CFs can be addressed to Jain's theory with all advantages of the related conclusions [17], in particular of the integer quantum Hall effect in the rest-field, leading to hierarchy of FQHE $[16,17]$.

One can thus summarize why 2D-charged particles must be associated with classical multi-loop braids for fields corresponding to fractional filling of LL. For $\nu=1$ one has $R_{\mathrm{c}}=R_{0}$ (where $R_{\mathrm{c}}$ is the cyclotron radius, $\pi R_{\mathrm{c}}^{2} B=\frac{h c}{e}$ and $2 R_{0}$ is the separation of particles, adjusted to the density and fixed by the short-range part of the Coulomb repulsion, $\left.\pi R_{0}^{2}=\frac{S}{N}\right)$. For $\nu<1$ the radius of cyclotron trajectory without additional loops $R_{\mathrm{c}}<R_{0}$, and then $R_{\mathrm{c}}$ is too short for particle interchange along these trajectories. Additional loops can, however, enhance $R_{\mathrm{c}}$ and again allow interchanges, since for $p$-loop cyclotron trajectories $\frac{h c}{e}=\pi R_{\mathrm{c}}^{2} \frac{B}{p}$, and $R_{\mathrm{c}}$ grows in comparison to single-loop trajectories; for $\nu=\frac{1}{p}$, again $R_{\mathrm{c}}=R_{0}$, though the external field is $p$ times bigger than for $\nu=1$ (at constant $N$ ). The fictitious fluxes of Jain's CFs played actually the similar role leading to an increase of cyclotron radius 
in the reduced resultant field. One can conclude thus that for $\nu=1$ the cyclotron trajectories are single-loop and braids are generated by $b_{i}^{(p=1)}=$ $\sigma_{i}$, while for $\nu=\frac{1}{p}, p>1$, the cyclotron trajectories must be multi-loop, simultaneously resulting in braids generated by $b_{i}^{(p)}=\sigma_{i}^{p}$.

Note finally that for a fixed magnetic field orientation the one-side cyclotron rotation is admitted, thus the cyclotron subgroup should be confined to its semigroup structure only. It does not cause, however, any perturbations of relevant 1DURs of cyclotron subgroups, which are crucial for identification of composite particles.

The additional loops associated with the appropriate subgroup generators lead to the phase shifts for particle interchanges, just as for Jain's CFs and permit corresponding Laughlin-type function requirements to be satisfied. These loops replace the fictitious screening fluxes. Note once more that multi-loop trajectories (similarly as single-loop ones) have only meaning in classical braid terms. Quantum particles do not traverse any trajectories, also any multi-loop cyclotron trajectories. The corresponding wave functions transform, however, in an agreement with 1DURs of the brad group or of the subgroup $[19,20]$, resulting in appropriate statistics behavior.

Let us emphasize that though CFs actually are not compositions of particles and vortices, we have not modified the original name "composite fermions." Moreover, we use the similar name 'composite anyons' for particles associated with fractional 1DURs (i.e., with fractional $p \alpha$ in equation (3.3)) of the cyclotron subgroup instead of the full braid group. The phase shift $\theta$ can be calculated as the Berry's phase along closed trajectory in configuration space for model multi-particle wave function corresponding to lowenergy excitations above the ground state at fractional filling of LL. These excitations - quasiparticles/quasiholes were traditionally associated with anyons in the case of a fractional Berry's phase. It is, however, clear that it is impossible to distinguish between fractional $\theta$ and $p \alpha-$ both these phase shifts can be the same fraction. As considered quasiparticles/quasiholes are excitations at the magnetic field presence, thus these states should be rather associated with cyclotron braids 1DURs, and therefore are composite anyons and not ordinary anyons, as previously regarded. This change, anyons for composite anyons, would result in convenient for QIP more dense relevant MDURs corresponding to braid subgroup instead of the full braid group.

\subsection{A role of the short-range part of the Coulomb interaction}

The crucial character of the short-range part of the Coulomb interaction for Laughlin correlations is visible from the fact that the Laughlin function is an 
accurate ground state wave function at $\frac{1}{p}$ LL filling, if to confine the Coulomb interaction represented by the so-called Haldane's pseudopotential [6,24], $V=\sum_{i>j} \sum_{m}^{\infty} V_{m} P_{m}^{i j},\left(P_{m}^{i j}\right.$ is the projector on the states of $i$ th and $j$ th particles with relative angular momentum $m$ ), to the components $V_{m}$, with $m=1, \ldots, p-2$ only. These $V_{m}$ terms, the Coulomb interaction energy of an particle pair with relative angular momentum $m$, contribute the shortrange part of the interaction of electrons, and the remaining terms - longrange interaction tail, corresponding to greater particle separation, i.e., with $m=p, \ldots$, do not influence strongly the Laughlin function [6,24,25]. The Laughlin correlations are associated with the incompressible states which correspond to discrete spectrum of Coulomb interaction projected on LL states, i.e., interaction expressed in terms of Haldane's pseudopotential with components assigned by relative angular momentum of particle pairs. This property, essential for FQHE, was even named by Laughlin as 'a quantization of particle separation' $[6,8]$. Quantization of the Coulomb interaction after projection on relative angular momentum of particle pairs in LL Hilbert subspace results in incompressible FQHE states numbered by integers (eigenvalues of relative angular momentum of particle pairs), the same which occur in the Laughlin functions (the exponent in the Jastrow polynomial). It is important to note that according to an attitude to FQHE using Haldane's pseudopotential (confined to the short-range part of the Coulomb repulsion), the Laughlin correlations revealed in the multi-particle wave function are unambiguous possibility for accurate ground-state at fractional LL filling, not only a variational result of the ground state modeling [6,24]. It supports an idea that Laughlin correlations are a fundamental topology-originated property of interacting charged 2D particles. One can thus expect that this Landau quantization behavior of interacting 2D-charged system must also manifest itself within braid group quantization approach to the same system, via the introduced cyclotron subgroup structure.

Since the Laughlin correlations can be expressed within CF approach, thus the Coulomb repulsion (the short-range part of Haldane's pseudopotential) is of a fundamental significance also for the CF construction. It should be, however, emphasized that the Coulomb interaction with the discrete spectrum, i.e., with separation by energy gaps the distinct relative angular momenta of particle pairs for sufficiently high magnetic field (noticeable via projection of the interaction on fractional filled LL as in the definition of Haldane's pseudopotential) does not play a role of standard dressing of particles with interaction, typical for quasiparticles in solids, just because the interaction has not a continuous spectrum in this projection.

An effective description of a local gauge field attached to particles is supplied by the Chern-Simons (Ch-S) field theory (chiral field, i.e., breaking 
time reversion and parity). This approach revived $[26,27]$ in the area of FQHE successfully describing particles with fluxes, in particular anyons and Jain's CFs [17]. It still, however, does not explain, what the spontaneously arising fluxes are.

It was demonstrated $[6,25]$ that the short-range part of the Coulomb interaction stabilizes $\mathrm{CFs}$ against action of $\mathrm{Ch}-\mathrm{S}$ field (its antihermitean term), which mixes states with distinct angular momenta within LL [25] in disagreement with the Jain's CF model in Ch-S field approach [17, 25]. The Coulomb interaction removes the degeneracy of these states and results in energy gaps which stabilize CF picture, especially effectively within the lowest LL. For higher LLs CFs are not so useful due to possible mixing between LLs induced by interaction [28].

The short-range part of the Coulomb interaction stabilizes CFs also in our description, similarly as it removes instability caused by Ch-S field for angular momentum orbits in LL [25]. Indeed, if the short-range part of the Coulomb repulsion was reduced, the separation of particles would not be rigidly kept (adjusted to a density only in average) and then another cyclotron trajectories, additional to those for fixed particle separation (multiloop at $\nu=\frac{1}{p}$ ), would be admitted, which violates the subgroup construction.

Thus the short-range part of the Coulomb interaction turns out to be crucial for $\mathrm{CF}$ formation in any description. Confining of the full braid group to the subgroup with multi-loop structure of cyclotron motion is justified only for particle separation adjusted to the double cyclotron radius. It is a role of the short-range part of the Coulomb repulsion which does not allow closer inter-particle separation than that which follows form the density. In this manner the short-range part of the Coulomb interaction is involved in the construction of the cyclotron braid structure. The long-range tail of the Coulomb interaction is left as a residual interaction of particles, which agrees with the Jain's model of weakly interacting CFs [16,17].

\section{Conclusions}

We have developed the braid group description for the case of $N$-charged 2D particle system at strong magnetic field presence, via definition of the cyclotron braid subgroups. This formalism allowed for interpretation of the Laughlin correlations of 2D-charged systems within the braid group approach to $N$ particle quantum systems. In this manner we formulated a new implementation of CFs employing braid group methods. Braid description of CFs was not previously established because of $2 \pi$ period of 1DURs. 
In the present paper we have avoided this problem via reduction of 1DURs to specially chosen braid subgroups selected in accordance with a 2D cyclotron motion. These cyclotron braid subgroups, generated by the new generators $b_{i}^{(p)}=\sigma_{i}^{p}\left(p=1,3,5, \ldots\right.$ enumerates a sort of composite anyons, $\sigma_{i}$ are generators of the full braid group), are separated braid objects which allow for distinguishing in statistics of CFs (with $p>1$ ) from ordinary fermions. It supports an idea that CFs are rightful 2D quantum particles which cannot be mixed with ordinary fermions, or with other sorts of CFs (although all correspond to antisymmetric functions). Distinguishing of CFs from fermions is important in particular for numerical diagonalization of interaction of CFs (not all antisymmetric functions can be admitted in diagonalization procedure, but only those which have the same phase shift due to particle interchanges, unless the mixing of various sorts of CFs took place [this is prohibited, similarly to the mixing of fermions and bosons in 3D]).

CFs turn out thus to be real 2D particles and not quasiparticles, i.e., they are not fermions dressed with interaction only, but are arranged as separate particles in topological terms. Identification of the special braid group object, the subgroup of the full braid group, associated with CFs, resolves also the problem of fictitious magnetic flux quanta, vortices, attached to these particles within the standard Jain's model. The Aharanov-Bohm phase shifts caused by hypothetical fluxes are replaced with the phase shifts due to additional $\frac{p-1}{2}$ loops during interchanges of particles (described in classical braid terms). These loops are an unavoidable property of interchanges of uniformly distributed (due to the Coulomb repulsion) 2D particles in a strong external magnetic field when ordinary cylotron radius is too short for particle interchanges (each particle traverses, in a classical braid picture, a closed $p$-loop cyclotron trajectory or in quantum language, it takes away $p$ quanta of the $B$ field flux; $p-1$ of them play the equivalent role as $p-1$ screening flux quanta attached to each CF in Jain's model).

The 1DURs, $b_{i}^{(p)} \rightarrow e^{i p \alpha}, \alpha \in(-\pi, \pi]$, of the cyclotron braid subgroups generated by $b_{i}^{(p)}(p$ - odd integer) supply, more generally, an implementation of composite anyons, including CFs of rank $p$, for $\alpha=\pi$. In particular, CFs (for $\alpha=\pi$ ) gain the phase shift $p \pi$ (due to the additional loops) the same as required by Laughlin-type correlations. The composite particles within the presented implementation are thus not connected with the full braid group but with their cyclotron subgroups. It makes CFs described rightfully with other types of $2 \mathrm{D}$ quantum particles within the uniform braid group approach, despite the $2 \pi$ period limitation for 1DURs.

An important role of the short-range part of the Coulomb interaction is indicated. This interaction fixes the inter-particle separation, (only in 
average determined by the planar density), which allows for definition of multi-loop cyclotron braid trajectories for particle interchanges in the case when single-loop cyclotron radius is too short in comparison to inter-particle separation, precluding particle exchanges along single-loop trajectories, as for $\frac{1}{p}$ LL filling. The additional loops reduce the total magnetic field flux and enhance the effective cyclotron radius, restoring possibility of particle interchanges. Thus multi-loop trajectories are unavoidable property of cyclotron braids leading, in a natural way, to the Laughlin correlations, without artificial constructions with vortices.

On the other hand, the cyclotron subgroups may have richer unitary representations, including MDURs, in comparison to the full braid group, which would result in more dense MDURs corresponding to composite nonAbelian anyons for possible QIP applications.

Supported by the Polish KBN project no N202-489939 and LFPPI Network.

\section{References}

[1] E. Spanier, Algebraic topology, Springer-Verlag, Berlin, 1966.

[2] J. S. Birman, Braids, links, and mapping class groups, Annals of Math. Stud. 82, Princeton University Press, Princeton, NJ, 1974.

[3] N. D. Mermin, The topological theory of defects in ordered media, Rev. Mod. Phys. 51 (1979), 591.

[4] D. C. Tsui, H. L. Störmer and A. C. Gossard, Two-dimensional magnetotransport in the extreme quantum limit, Phys. Rev. Lett. 48 (1982), 1559.

[5] S. Das Sarma and A. Pinczuk, Perspectives in quantum Hall effects: novel quantum liquids in low-dimensional semiconductor structures, Wiley, New York, 1997.

[6] R. E. Prange and S. M. Girvin, The quantum hall effect, SpringerVerlag, New York, 1990.

[7] R. B. Laughlin, Quantized motion of three two-dimensional electrons in a strong magnetic field, Phys. Rev. B27 (1983), 3383.

[8] R. B. Laughlin, Anomalous quantum Hall effect: an incompressible quantum fluid with fractionally charged excitations, Phys. Rev. Lett. 50 (1983), 1395.

[9] F. Wilczek, Fractional statistics and anyon superconductivity, World Scientific Publishing, Singapore, 1990. 
[10] Y. S. Wu, Flat bands and Wigner crystalization in honeycomb optical lattice, Phys. Rev. Lett. 52 (1984), 2103.

[11] E. Artin, Theory of braids, Ann. Math. 48 (1947), 101.

[12] L. Jacak, P. Sitko, K. Wieczorek and A. Wojs, Quantum hall systems: braid groups, composite fermions, and fractional charge, Oxford University Press, Oxford, 2003.

[13] T. D. Imbo, C. S. Imbo and E. C. G. Sudarshan, Identical particles, exotic statistics and braid groups, Phys. Lett. B234 (1990), 103.

[14] T. Einarsson, Fractional statistics on a torus, Phys. Rev. Lett. 64 (1990), 1995.

[15] C. Nayak, S. H. Simon, A. Stern, M. Freedman and S. Das Sarma, Nonabelian anyons and topological quantum computation, Rev. Mod. Phys. 80 (2008), 1083.

[16] J. K. Jain, Composite-fermion approach for the fractional quantum Hall effect, Phys. Rev. Lett. 63 (1989), 199.

[17] O. Heinonen, Composite Fermions, World Scientific Publishing, Singapore, 1998.

[18] B. I. Halperin, P. A. Lee and N. Read, Theory of the half-filled Landau level, Phys. Rev. B47 (1993), 7312.

[19] T. D. Imbo and E. C. G. Sudarshan, Inequivalent quantizations and fundamentally perfect spaces, Phys. Rev. Lett. 60 (1988), 481.

[20] E. C. G. Sudarshan, T. D. Imbo and T. R. Govindarajan, Configuration space topology and quantum internal symmetries, Phys. Lett. B213 (1988), 471.

[21] A. Kitaev, Quantum computations: algorithms and error correction, Russian Math. Survey 52(61) (1997), 1191; A. Kitaev, Fault-tolerant quantum computation by anyons, Annals Phys. 303 (2003), 2; M. Freedman, A. Kitaev, M. Larsen and Z. Wang, Topological quantum computation, Bull. Amer. Math. Soc. (N.S.) 40 (2003), 31.

[22] M. A. Nielsen and I. L. Chuang, Quantum computation and quantum information, Cambridge University Press, Cambridge, 2000.

[23] J. S. Xia, W. Pan, C. L. Vicente, E. D. Adams, N. S. Sullivan, H. L. Störmer, D. C. Tsui, L. N. Pfeiffer, K. W. Baldwin and K. W. West, Electron correlation in the second Landau level: a competition between many nearly degenerate quantum phases, Phys. Rev. Lett. 93 (2004), 176809; E. H. Rezayi and N. Read, Non-abelian quantized Hall states of electrons at filling factors 12/5 and 13/5 in the first excited Landau level, Phys. Rev. B79 (2009), 075306.

[24] F. D. M. Haldane, Fractional quantization of the Hall effect: a hierarchy of incompressible quantum fluid states, Phys. Rev. Lett. 51 (1983), 605. 
[25] T. Morinari, Composite fermion pairing theory in single-layer systems, Phys. Rev. B62 (2000), 15903.

[26] A. L. Fetter, C. B. Hanna and R. B. Laughlin, Random-phase approximation in the fractional-statistics gas, Phys. Rev. B39 (1989), 9679.

[27] A. Lopez and E. Fradkin, Fractional quantum Hall effect and ChernSimons gauge theories, Phys. Rev. B44 (1991), 5246.

[28] T. Sbeouelji and N. Meskini, Stability of the fractional quantum Hall effect in higher Landau levels for composite fermions carrying four vortices, Phys. Rev. B64 (2001), 193305. 
